\title{
Reflets
}

Revue ontaroise d'intervention sociale et communautaire

\section{Troubles de la communication et obstacles au travail : les défis des personnes et des organisations}

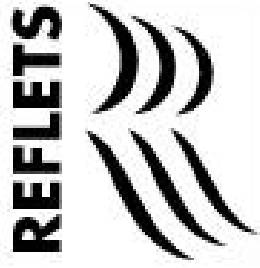

\section{Jacques Barrette, Chantal Laroche et Linda J. Garcia}

Volume 4, numéro 2, automne 1998

Personnes vivant avec une incapacité

URI : https://id.erudit.org/iderudit/026217ar

DOI : https://doi.org/10.7202/026217ar

Aller au sommaire du numéro

Éditeur(s)

Reflets : Revue ontaroise d'intervention sociale et communautaire

ISSN

1203-4576 (imprimé)

1712-8498 (numérique)

Découvrir la revue

Citer cet article

Barrette, J., Laroche, C. \& Garcia, L. J. (1998). Troubles de la communication et obstacles au travail : les défis des personnes et des organisations. Reflets, 4(2), 47-62. https://doi.org/10.7202/026217ar

Tous droits réservés (C) Reflets : Revue ontaroise d'intervention sociale et communautaire, 1998
Ce document est protégé par la loi sur le droit d'auteur. L'utilisation des services d'Érudit (y compris la reproduction) est assujettie à sa politique d'utilisation que vous pouvez consulter en ligne.

https://apropos.erudit.org/fr/usagers/politique-dutilisation/ 


\section{Troubles de la communication et obstacles au travail : les défis des personnes et des organisations}

Jacques Barrette

Faculté des sciences de l'administration et

Chantal Laroche et Linda J.Garcia,

Programme d'audiologie et d'orthophonie, Université d'Ottawa

\section{Introduction}

"...nous savons qu'au cours des dix prochaines années, le marché du travail passera d'un marché secondaire (industrie) à un marché d'emplois basé sur les services et les technologies de communication.»
La communication est une nécessité de la vie de tous les jours et sera de plus en plus omniprésente dans le milieu du travail. En effet, les changements technologiques ont forcé le secteur manufacturier à compter davantage sur la mécanisation de leurs opérations. Par conséquent, on a assisté à une diminution drastique de la création des emplois dans ce milieu. Cette révolution technologique force les individus à se recycler dans le secteur tertiaire où la communication est un outil de travail prépondérant. En effet, nous savons qu'au cours des dix prochaines années, le marché du travail passera d'un marché secondaire (industrie) à un marché d'emplois basé sur les services et les technologies de communication (Kutscher 1992). En l'an 2000, le domaine des services fournira $90 \%$ de tous les nouveaux emplois et une grande partie de ceux-ci sera reliée aux services à la clientèle (Carey et Franklin 1992). Ce milieu doit et devra gérer l'incapacité de façon efficace. Actuellement, dans ce milieu, plusieurs nouveaux cas d'incapacité voient le jour en raison des demandes particulières qu'imposent les emplois de service ${ }^{1}$. 
On voit apparaître des problèmes de santé liés à l'utilisation intensive de l'ordinateur (ex. vision). Parmi les troubles pouvant créer une incapacité, on retrouve les troubles de la communication.

Un trouble de la communication est défini comme «une incapacité dans l'habileté à recevoir, transmettre, traiter et comprendre les concepts verbaux et non verbaux ainsi que les systèmes de symboles graphiques» (American Speech-LanguageHearing Association 1995). Un trouble de la communication peut se manifester au niveau de l'audition, du langage ou de la parole. Son degré de sévérité peut s'étendre de léger à profond et le trouble peut être congénital ou acquis. En 1986, un sondage indiquait que $30 \%$ des Canadiens ayant des incapacités avaient des difficultés reliées au langage (6\%) et à l'audition (24\%) (Statistics Canada 1990).Toutefois, le sondage de 1986 n'identifiait pas nécessairement les individus ayant des troubles de la communication plus subtils, tels que des troubles de bégaiement et de compréhension non reliés à l'audition découlant d'un accident cérébro-vasculaire ou d'un traumatisme crânien (Johnson 1987). Tous ces groupes de personnes peuvent vivre des situations difficiles de réintégration au travail. Une incapacité sur ce plan est particulièrement problématique puisque dans le milieu des services, la communication est sans contredit l'un des principaux outils de travail. Compte tenu de la prépondérance de la communication dans ce secteur, il est prévu que les personnes atteintes de troubles de la communication éprouveront une difficulté supplémentaire à réintégrer leur emploi ou le marché du travail et pourraient être particulièrement défavorisées lors de l'administration des programmes d'invalidité. Le milieu des services risque ainsi de devenir une source de frustration, de stress et créer des situations de handicap et de la discrimination.

\section{Un défi à partager}

En milieu de travail, les défis auxquels doivent faire face les individus ayant un trouble de la communication sont 
"...plusieurs troubles de la communication sont invisibles au premier abord, tel que par exemple les troubles de l'audition. Cette invisibilité fait en sorte qu'ils ne sont pas pris en considération lors de l'adaptation du poste de travail au même titre qu'une incapacité physique requérant par exemple un fauteuil roulant.» particulièrement nombreux et importants. D'une part, plusieurs troubles de la communication sont invisibles au premier abord, tel que par exemple les troubles de l'audition. Cette invisibilité fait en sorte qu'ils ne sont pas pris en considération lors de l'adaptation du poste de travail au même titre qu'une incapacité physique requérant par exemple un fauteuil roulant. D'autre part, en raison de cette invisibilité mais aussi du peu de recherche dans le domaine, ils sont mal connus des professionnels de la gestion des ressources humaines qui ont la responsabilité de l'embauche, de l'organisation du travail ou de la gestion des programmes d'invalidité (disability management). Les personnes avec un trouble de la communication devront donc faire face au défi de trouver, souvent par eux-mêmes et parfois avec l'aide des intervenants professionnels (orthophonistes et audiologistes), des stratégies pour amoindrir ou contourner les obstacles qui les empêchent d'être membre actif et à part entière du monde du travail. Toutefois, le défi de la réintégration au travail ne repose pas uniquement sur les épaules de la personne atteinte de troubles de la communication ou des intervenants professionnels du domaine. Les employeurs ont aussi un rôle crucial sur ce plan. Ils ont la responsabilité et le devoir légal de créer un environnement de travail exempt de barrières discriminantes en adaptant leurs processus de gestion ainsi que les postes de travail en y apportant des accommodations raisonnables. Cette responsabilité est d'autant plus grande qu'elle implique des coûts importants si elle est ignorée ou mal gérée.

En effet, compte tenu de leur responsabilité légale, les employeurs doivent assumer une grande partie des coûts reliés à l'invalidité professionnelle (ex. primes d'accident de travail) et non professionnelle (ex. programmes d'assurances invalidités court terme et long terme). En 1996, au Canada, la compensation payée aux personnes ayant des incapacités permanentes s'est chiffrée à 1,8 milliard de dollars alors qu'un autre 1,9 milliard de dollars ont été payés aux personnes atteintes d'incapacités temporaires. Pour leur part, les coûts des programmes de réadaptation professionnelle pour les personnes atteintes d'incapacités se sont chiffrés à 408 millions de dollars. Les coûts reliés à la gestion des invalidités dans les organisations continuent d'augmenter et 
"Des effets négatifs tels la détérioration de la santé du titulaire, la baisse de productivité, l'insatisfaction des collègues et $d u$ gestionnaire, le renforcement des préjugés sur la non productivité des personnes avec une incapacité ne sont que quelques conséquences d'une gestion inefficace des invalidités.» affectent l'actif des employeurs, les risques de l'assureur et la personne avec l'incapacité. Les coûts passent souvent aux consommateurs sous la forme de prix plus élevés pour les biens et services. On estime que les coûts de la gestion des invalidités ont augmenté de $35 \%$ depuis 1986. Dans la société, compte tenu de l'importance des troubles de la communication et de la prépondérance de la communication dans les emplois actuels, il est permis de penser qu'une proportion importante de ces coûts sont et seront affectés à la gestion des invalidités liées aux troubles de la communication. Toutefois, le fait de ne pas réintégrer des personnes avec incapacité n'a pas seulement des conséquences monétaires importantes. Des effets négatifs tels la détérioration de la santé du titulaire, la baisse de productivité, l'insatisfaction des collègues et du gestionnaire, le renforcement des préjugés sur la non productivité des personnes avec une incapacité ne sont que quelques conséquences d'une gestion inefficace des invalidités. L'absence de programme efficace de gestion créerait aussi un effet discriminatoire contre certains groupes selon leur type d'incapacité (Rice et Kroll 1994).

\section{Comprendre les défis vécus au travail}

Depuis quelques années, plusieurs chercheurs ont tenté de mieux comprendre les défis vécus au travail par les personnes atteintes de troubles de la communication. Plusieurs auteurs affirment qu'un grand nombre de facteurs auxquels sont confrontées les personnes avec troubles de la communication affecteraient leurs chances de réinsertion à l'emploi ou la possibilité d'être considérées équitablement dans les processus organisationnels tels que la dotation et l'évaluation. Cette littérature suggère que ces obstacles sont liés à l'environnement de travail, aux exigences des emplois, aux conditions de travail, aux rapports psychosociaux et à certaines politiques et systèmes organisationnels de ressources humaines. 


\section{Environnement de travail, exigences des emplois et conditions de travail}

"Bien qu'il n'y ait aucune raison de croire que les personnes atteintes de perte auditive ont moins d'habiletés à se trouver un emploi, des études indiquent que celles-ci ont plus de difficultés à conserver leur emploi et sont moins satisfaites en ce qui concerne les caractéristiques intrinsèques $d u$ travail...»
Bien qu'il n'y ait aucune raison de croire que les personnes atteintes de perte auditive ont moins d'habiletés à se trouver un emploi, des études indiquent que celles-ci ont plus de difficultés à conserver leur emploi et sont moins satisfaites en ce qui concerne les caractéristiques intrinsèques du travail (ex. variété, autonomie, possibilité de carrière, etc.) (Kyle et Wood 1985; Thomas et Herbst 1980). Ces recherches suggèrent que certaines caractéristiques reliées à l'environnement en général, aux tâches de l'emploi et aux conditions de travail sont peu adaptées à leurs troubles, et créeraient un obstacle à leur satisfaction et à leur adaptation, ce qui entraînerait des départs prématurés.

Ainsi, au niveau de l'environnement de travail, on peut penser que l'utilisation massive d'équipements de communication (téléphone, répondeur automatique, ordinateur parlant) et l'aménagement physique des lieux de travail (ex. bureau à aire ouverte) créent des barrières qui nuisent à l'intégration au travail. Sur ce plan, Rolland et Belin (1991) pensent que des facteurs extrinsèques (ex. manque d'adaptation des milieux de travail) compromettent le retour au travail des personnes atteintes de troubles du langage. En ce qui a trait à l'emploi, les nouvelles formes d'organisation du travail (ex. groupe autonome et partiellement autonome) pourraient s'avérer des obstacles susceptibles de rendre difficile l'adaptation au travail des personnes avec des troubles de la communication. Par exemple, un travail en groupe qui exigerait de traiter simultanément des informations verbales en présence de bruit dans une situation stressante pourrait s'avérer une barrière pour plusieurs personnes avec un trouble de la communication.

\section{Rapports psychosociaux}

Les interactions avec les clients, les collègues et le superviseur peuvent s'avérer une difficulté pour les personnes atteintes de certains troubles et créer des barrières sur le plan social. Selon Brown etYule (1983), la communication servirait deux fonctions : 
"Les interactions avec les clients, les collègues et le superviseur peuvent s'avérer une difficulté pour les personnes atteintes de certains troubles et créer des barrières sur le plan social.» l'une transactionnelle et l'autre interactionnelle. L'expression du contenu et l'échange d'information répondraient à la fonction transactionnelle. Par contre, la fonction interactionnelle viserait l'établissement et le maintien des relations sociales. Une personne ayant un trouble de la communication se verrait donc privé de ses deux fonctions. Dans une étude récente, Hétu, Getty et Waridel (1994) ont montré que les sujets ayant des pertes auditives craignaient d'être perçus négativement et tendaient à s'isoler pour cacher leur incapacité. Rice (1995) a montré que l'aliénation sociale était un facteur affectant l'accessibilité à l'emploi pour les personnes ayant un bégaiement. Pour leur part, Rolland et Belin (1991) ont montré que des obstacles reliés à l'acceptation sociale (ex. manque de soutien des collègues de travail) compromettaient le retour au travail des personnes vivant des troubles du langage suite à un accident cérébro-vasculaire.

\section{Organisation et systèmes de gestion des ressources humaines}

Les biais négatifs défavorables aux personnes atteintes de troubles de la communication peuvent être particulièrement importants lors de l'entrevue de sélection. Nous savons que les premières impressions négatives, de la part d'un interviewer non averti, orientent un biais défavorable qui affectera la décision finale d'embauche (Jackson, Peacock et Smith 1980). Les incapacités, même lorsqu'elles sont indépendantes des exigences d'emploi, peuvent facilement être perçues comme des traits non désirés et entraîner les erreurs de première impression et de halo défavorables à l'individu. Dans ce sens, les recherches de Barrette et Haccoun (1995) ont montré que les évaluateurs orientent facilement leurs jugements vers la négative lorsque les traits sont considérés

"...les troubles de la communication sont plus critiques que les autres types d'incapacités, car la première impression est souvent basée sur l'habileté à communiquer.» socialement indésirables. Pour leur part, Rolland et Belin (1991) soutiennent que lors du processus de sélection, les troubles de la communication sont plus critiques que les autres types d'incapacités, car la première impression est souvent basée sur l'habileté à communiquer. Plusieurs études indiquent que la perception des personnes ayant des troubles de la communication par les gens de leur entourage tendrait à être négative (Beukelman, Kraft et Freal 1985; Hétu, Getty et Waridel 1994; Kalinowski, Stuart et Armson 1996; McKinnon, Hess et Landry 1986). 
Un autre obstacle à l'embauche et à l'insertion au marché du travail semble être relié au type d'instruments psychométriques utilisés pour choisir les candidats. Nous savons que les tests d'aptitudes cognitives pour évaluer le potentiel des candidats lors de la sélection sont largement utilisés au Canada (Gertkate, Hausdorf et Cronshaw 1992). Ce type de tests de sélection utilise de l'information dite verbale (ex. grammaire, compréhension verbale), c'est-à-dire un des types d'information qui peut être difficile à traiter par certaines personnes ayant un trouble de la communication, et ce en raison même de leur incapacité. Il est raisonnable de penser que leur performance à ces tests pourrait en être affectée, car les personnes avec un trouble de la communication pourraient avoir une probabilité plus grande d'être évaluées sur leurs incapacités plutôt que sur les dimensions requises par l'emploi. Cette remarque est également plausible en ce qui concerne l'entrevue de sélection. De plus, l'absence de validation des tests d'aptitudes cognitives auprès de cette population risque d'avoir un impact défavorable sur ce groupe. Sans instrument de mesures approprié, adapté et validé auprès des individus ayant des incapacités de communication, le jugement demeure basé sur des biais psychométriques.

Une fois dans un emploi, ces personnes sont confrontées au système de promotions internes. En effet, les recherches actuelles indiquent que la diminution des chances d'avancement serait un problème important auquel font face ces personnes. Les études indiquent que, pour ces individus, le statut d'emploi et les opportunités d'avancement seraient compromis. Ainsi, MacLeodGallinger (1992) a trouvé que les femmes atteintes de surdité étaient $33 \%$ moins employées que les autres femmes. Par ailleurs, Felsenfeld, Broen et McGue (1994) ont montré que le statut d'emploi était plus bas pour les personnes avec des troubles phonologiques. Pour leur part, Rice et Kroll (1994) indiquaient que $70 \%$ des sujets interrogés se voit imposer des limites d'emploi en raison de leur problème de bégaiement. En dehors des limites fonctionnelles des individus, certaines raisons liées à la gestion des ressources humaines pourraient expliquer ces résultats. Par exemple, la formation fournie par les entreprises pourrait ne pas être adaptée aux individus avec troubles de la communication et 
«Toutefois, pour que ces programmes soient efficaces, ils doivent s'appuyer sur un diagnostic de l'incapacité, sur une connaissance approfondie de ces barrières et de leur cause ainsi que sur l'identification des stratégies d'interventions adaptées au milieu.» ainsi réduire leur chance d'acquérir des compétences additionnelles nécessaires pour accéder à des emplois supérieurs. Il est possible aussi que les décisions de promotion sur la base de la compétence soient masquées par le trouble de la communication au même titre que les décisions lors de la sélection.

Les promotions sont souvent reliées au résultat de l'évaluation du rendement. Nous savons que le processus d'évaluation du rendement est facilement entaché d'erreur de jugement provenant des idées préconçues (Barrette et Haccoun 1995). Nous savons aussi que les caractéristiques signalétiques telles que l'âge (Arvey, Miller, Gould et Burch 1987), la race (McDonald et Hakel 1985), le sexe (Gorman, Clover et Doherty 1978; Heilman et Martell 1986) et l'histoire personnelle du sujet (London et Hakel 1974) influencent le jugement lors de l'évaluation. À ce jour, aucune recherche n'a vérifié l'impact des troubles de la communication sur les résultats de l'évaluation. On peut émettre l'hypothèse d'une relation désavantageuse sur ce plan pour les personnes avec troubles de la communication.

Plusieurs des obstacles soulevés par ces auteurs pourraient être amoindris ou même éliminés par des programmes de gestions des invalidités. La gestion des incapacités est définie comme un processus actif pour minimiser l'impact d'une incapacité sur la capacité des individus à concurrencer dans le monde du travail (Shrey 1997). C'est un processus proactif qui promeut des stratégies de prévention, de concept de traitement de réadaptation et un programme de retour au travail conçu pour contrôler les coûts personnels et économiques de l'incapacité en milieu de travail. Toutefois, pour que ces programmes soient efficaces, ils doivent s'appuyer sur un diagnostic de l'incapacité, sur une connaissance approfondie de ces barrières et de leur cause ainsi que sur l'identification des stratégies d'interventions adaptées au milieu.

\section{Les objectifs de la recherche}

Dans le cadre d'un projet de recherche s'échelonnant sur trois ans, les auteurs actuels ont entrepris: 
a) de déterminer avec plus de précision les facteurs environnementaux (ex. équipement, bruit) et organisationnels (ex. processus de dotation, politique de gestion) menant à des situations de handicaps en milieu de travail pour les personnes avec troubles de la communication, c'est-à-dire les obstacles qui pourraient les empêcher d'être embauchées ou de s'intégrer au travail;

b) d'identifier les stratégies d'adaptation pouvant être mises de l'avant par l'individu, l'intervenant et l'employeur afin de faciliter l'intégration au travail de ces personnes.

Ce projet impliquait d'interviewer 78 individus avec troubles de la communication, 28 professionnels intervenants et 22 employeurs des secteurs privé et public. Ces personnes, réunies en groupe de travail (focus groups), ont identifié un très grand nombre d'obstacles qui nuisent ou empêchent l'intégration ou la réintégration au travail ainsi qu'une multitude de stratégies ou moyens concrets pour contourner ou amoindrir ces barrières.

Conformément aux objectifs du projet, la première tâche demandée aux participants a été d'identifier, suite à leur expérience vécue, les obstacles en milieu de travail qui sont susceptibles de mener à des situations de communication difficiles et empêchent l'embauche ou l'intégration des personnes avec troubles de la communication ${ }^{2}$. À l'aide de la technique du groupe nominal (Brunelle, Drouin, Godbout et Tousignant 1988), la deuxième tâche a permis de distinguer de l'ensemble des obstacles répertoriés ceux ayant le plus d'importance. Enfin, la troisième tâche consistait à demander aux participants de trouver toutes les stratégies ou les moyens possibles pouvant exister ou à créer pour surmonter les obstacles retenus à l'étape précédente.

Six familles de troubles de la communication ont été considérées dans l'étude :

1) les troubles de la parole (ex. dysarthrie) regroupent les problèmes associés à la clarté de la parole et peuvent se manifester par une voix nasillarde, une parole monotone, par 
«Six familles de troubles de la communication ont été considérées dans

l'étude :

1) les troubles de la parole...

2) le bégaiement...

3) la laryngectomie totale...

4) l'aphasie...

5) les troubles d'audition...

6) les troubles de la voix..." la difficulté à produire des sons clairs, de longues phrases et la nécessité pour certains d'utiliser un tableau de communication électronique,

2) le bégaiement est un trouble qui affecte le débit de la parole et s'observe, entre autres, par la répétition de parties de mots, le prolongement de sons, l'insertion de sons, de syllabes ou de phrases,

3) la laryngectomie totale implique l'absence de cordes vocales et l'utilisation d'une méthode alternative pour communiquer (ex. larynx artificiel),

4) l'aphasie est un trouble de langage associé à une lésion cérébrale, suite par exemple à un accident cérébro-vasculaire et peut entraîner, entre autres, une difficulté à trouver les mots, la substitution de mots, une parole de style télégraphique, des problèmes de lecture et d'écriture,

5) les troubles d'audition réferent aux problèmes de réception d'un message auditif et peuvent entraîner principalement une difficulté à entendre certains sons, à comprendre la parole dans le bruit, à localiser les sources sonores et peuvent être accompagnés de bourdonnements d'oreille,

6) les troubles de la voix regroupent les problèmes avec la qualité de la voix tels qu'une voix rauque, trop aspirée, des pertes de voix ou une voix avec un timbre qui ne convient ni à l'âge ni au sexe (trop aiguë ou trop grave).

Une analyse itérative impliquant toute l'équipe de recherche a permis de classifier les obstacles et les stratégies sous des facteurs plus généraux. Plusieurs des résultats obtenus confirment le point de vue des divers auteurs sur le sujet.Toutefois, plusieurs obstacles identifiés sont totalement nouveaux et n'ont jamais été soulevés dans les écrits précédents. Finalement, les stratégies proposées par les participants représentent autant de moyens concrets que les personnes avec troubles de la communication, les intervenants et particulièrement les employeurs peuvent utiliser pour réduire les barrières à l'emploi pour les personnes vivant un trouble de la communication. Pour les employeurs ces informations sont autant d'éléments qui peuvent contribuer à rendre leur programme de gestion des invalidités plus efficaces. 


\section{Identification des obstacles et des stratégies}

"Ainsi, les exigences

de certaines conditions

de travail telle que la

nécessité de répondre à des situations urgentes, certaines

caractéristiques de

l'environnement

physique (ex. bruit, qualité de l'air),

l'usage de technologies

orales auditives (ex. répondeur) et le travail en groupe sont des exemples de situations qui, si elles ne sont pas adaptées, peuvent créer une barrière à la réintégration des personnes avec troubles de la communication.»
Les résultats montrent qu'en dehors des obstacles reliés à l'intégrité du système (limites fonctionnelles imposées par le trouble) et aux comportements (limites que s'imposent le sujet), les obstacles liés à la nature du travail sont particulièrement importants. Ainsi, les exigences de certaines conditions de travail telle que la nécessité de répondre à des situations urgentes, certaines caractéristiques de l'environnement physique (ex. bruit, qualité de l'air), l'usage de technologies orales auditives (ex. répondeur) et le travail en groupe sont des exemples de situations qui, si elles ne sont pas adaptées, peuvent créer une barrière à la réintégration des personnes avec troubles de la communication.

Les attitudes et les comportements sociaux des autres personnes représenteraient une autre gamme de difficultés importantes à surmonter. Plusieurs des obstacles qui ont été identifiés sous ce thème sont reliés aux attitudes et comportements des clients, des collègues et des superviseurs. Ces données supportent les résultats de d'autres recherches qui montrent que la perception négative de l'entourage rend difficile l'accessibilité ou la progression en emploi pour les personnes avec troubles de la communication (Beukelman, Kraft et Freal 1985; Hétu, Getty et Waridel 1994; Rolland et Belin 1991).

Un autre facteur soulevé touche aux processus de dotation, plus spécifiquement les tests de sélection (incluant les critères d'embauche) et l'entrevue de sélection. Ces résultats supportent le point de vue d'autres chercheurs qui soutiennent que certains tests de sélection, particulièrement les tests d'aptitudes, sont inadaptés lorsqu'ils sont appliqués aux personnes avec troubles de la communication (Nester 1984). Pour sa part, les attitudes et comportements des interviewers lors de l'entrevue de sélection sont des barrières qui peuvent être déterminantes sur l'accessibilité à l'emploi.

Un autre facteur mentionné concerne la capacité de l'organisation à intervenir. Cette catégorie réfere à l'aptitude de 
l'organisation à adapter les postes de travail ou à mettre en place des actions ou programmes pour faciliter la réintégration des personnes avec troubles de la communication. Les résultats obtenus indiquent que cette capacité peut être limitée par des aspects financiers ou encore par l'orientation corporative habituellement reflétée dans les valeurs de l'organisation. Il est certain que les coûts qu'engendre l'adaptation des tâches peuvent pour certaines entreprises constituer un frein à l'accommodation du poste de travail. Toutefois, lorsque l'on sait que la plupart des accommodations coûtent moins de $500 \$$ (Commission canadienne des droits de la personne 1997), il est permis de penser que cette barrière pourrait être amoindrie avec une meilleure information aux responsables de la gestion des ressources humaines.

La pression pour une productivité élevée créerait une autre limite pour les divers groupes consultés. En effet, la charge de travail et les exigences de performance maximum réduiraient grandement la probabilité de succès d'un retour au travail pour ces personnes. Finalement, le manque de sensibilisation des employeurs, le peu de programmes de support socio-économiques ainsi que les réalités du marché de l'emploi tels que la tendance à la réduction des effectifs dans les organisations, sont d'autres facteurs qui limitent l'accès au groupe concerné.

Cette étape a permis de développer une grille permettant de diagnostiquer rapidement les principaux facteurs pouvant limiter l'embauche et l'intégration des personnes qui ont des troubles de la communication ou de toutes personnes qui a des difficultés importantes à communiquer. Cette grille de classification pourrait également servir de mesure pour évaluer les programmes d'invalidité sur le plan de la communication, incluant les aspects physiques du poste de travail.

\section{Un défi pour les employeurs}

Les résultats obtenus, dans la troisième phase de la cueillette, indiquent que plusieurs des obstacles à l'intégration au travail 
pourraient être contournés ou éliminés par des stratégies plus efficaces adoptées par l'individu, les intervenants ou l'employeur. Lors de cette phase, un grand nombre de stratégies ont été proposées par les participants à l'étude. Certaines de ces stratégies s'adressent directement aux personnes avec troubles de la communication et concernent des changements d'attitudes et de comportements initiés par ces dernières pour surmonter le handicap. Une meilleure préparation pour faire face à l'entrevue de sélection, la création d'un réseau de contact, le développement de certaines capacités dans des domaines cibles, la pratique de certaines techniques de relaxation en sont quelques exemples.

Toutefois, un grand nombre de ces stratégies visent spécifiquement les employeurs. Par exemple, certaines touchent la sensibilisation des superviseurs et des collègues, ainsi que l'aide et le soutien spécifique qu'ils peuvent apporter lors de la réintégration au travail de la personne avec troubles de la communication. Plusieurs des stratégies concernent l'adaptation ou la modification du poste de travail tel que le remplacement de certaines tâches qui exigent une communication rapide par l'assignation à des tâches à haut potentiel de productivité pour ces personnes. D'autres stratégies ont trait à l'organisation du milieu physique tels que l'emplacement du bureau dans un endroit moins bruyant ou achalandé. Des nombreuses propositions visent

«Par exemple, on demande aux employeurs d'effectuer des analyses de tâches qui tiennent compte des particularités de la personne, et que les attentes de rendement soient établies progressivement afin que la personne puisse s'adapter à un nouveau poste.» l'adaptation de technologies existantes tels que le téléphone, le courrier électronique, les boîtes vocales, la vidéoconférence, l'audioconférence, etc. Plusieurs suggestions visent l'adaptation de certains systèmes de gestion des ressources humaines. Par exemple, on demande aux employeurs d'effectuer des analyses de tâches qui tiennent compte des particularités de la personne et que les attentes de rendement soient établies progressivement afin que la personne puisse s'adapter à un nouveau poste. Des suggestions portent également sur l'amélioration et la validation des outils de sélection utilisés lors de l'embauche et sur une adaptation de la formation donnée en entreprise. La formation du superviseur est également visée. Par exemple, cette formation devrait avoir comme objectif de l'aider à mieux comprendre le phénomène, mais aussi à mieux jouer son rôle d'entraîneur. 
Plusieurs stratégies diverses et spécifiques ont été suggérées telles que l'implication et le partenariat avec les associations et organismes communautaires, l'aménagement des salles de réunion et la création d'une base de données provinciale sur les emplois cibles.

\section{Conclusion}

«Les résultats de l'étude démontrent que le défi de la réintégration au travail est un défi qui doit être partagé entre les intervenants professionnels, les employeurs et les personnes vivant une incapacité.»
Les exigences de plus en plus grandes en compétence de communication feront en sorte que les personnes ayant des troubles de la communication risquent d'être systématiquement exclues du marché du travail si aucune action n'est prise pour réduire ou éliminer les obstacles auxquels elles sont confrontées. L'identification des obstacles et des stratégies est un premier pas concret dans ce sens. Les résultats de l'étude démontrent que le défi de la réintégration au travail est un défi qui doit être partagé entre les intervenants professionnels, les employeurs et les personnes vivant une incapacité. La grille de diagnostic qui en découle donne aux intervenants professionnels un outil pour ajuster leur intervention en fonction de la réalité du milieu de travail. Pour les employeurs, cet outil fournit des indications claires sur les changements raisonnables pouvant être apportées aux postes de travail. Cet outil spécifie également les stratégies à inclure dans les programmes de gestion des invalidités afin de les rendre plus efficaces. Pour les personnes vivant un trouble de communication, cette grille leur permettra d'anticiper les difficultés et d'y trouver de multiples moyens concrets pour les éliminer ou les contourner.

Ainsi, de la même façon que nous sommes devenus familiers avec les modifications architecturales associées aux rampes pour les fauteuils roulants, des modifications aux conditions de travail et des changements aux mentalités pourraient faciliter l'intégration ou la réintégration de ces ressources humaines. Cette recherche a permis d'explorer une réalité jusqu'ici pratiquement inconnue mais tout à fait réelle pour les centaines de milliers de personnes atteintes de troubles de la communication. 


\section{Bibliographie}

AMERICAN SPEECH-LANGUAGE-HEARING ASSOCIATION (1995). «ASHA's consumer conscience». ASHA Reports, May, 14.18.

ARVEY, R.D., H.E. MILLER, R. GOULD ET P. BURCH (1987). «Interview Validity for Selecting Sales Clerks», Personnel Psychology, no 40,1-13.

BARRETTE,J.ET P.HACCOUN. (1995). «La précision de l'évaluation du rendement: une vérification de la théorie de Wherry", Revue canadienne des sciences de l'administration, vol. 12, no 4, 325-339.

BEUKELMAN,D.R., G.H.KRAFT ET J.FREAL (1985). «Expressive communication disorders in persons with multiple sclerosis:A survey", Archives of Physical Medicine and Rehabilitation, vol. 66, 675-677.

BROWN, G. ET G.YULE (1983). Discourse analysis, Cambridge, England, Cambridge University Press.

BRUNELlE, J., D. DROUIN, P. GODBOUT ET M. TOUSIGNANT (1988). La supervision de l'intervention en activité physique, Boucherville, Gaëtan Morin éd.

CAREY, M.L. ET J. C. FRANKLIN (1992). In Outlook 1990-2005. BLS Bulletin 2402. Washington, DC:U.S. Department of Labor, Bureau of Labor Statistics.

COMMISSION CANADIENNE DES DROITS DE LA PERSONNE (1997).«Employeur sans obstacles», Internet: /barrier/index.htm.

FELSENFELD, S., P.A. BROEN ET M MCGUE (1994). «A 28-year follow-up of adults with a history of moderate phonological disorder: educational and occupational results", Journal of Speech and Hearing Research, no 37, 1341-1353.

FOUGEYROLLAS, P., R. CLOUTIER, H.BER GERON,J.CÔTÉ, M. CÔTÉ ET G. ST-MICHEL (1996). Révision de la proposition québécoise de classification: Processus de production du handicap. Réseau international sur le processus de production du handicap.

GARCIA, BARRETTE ET C. LAROCHE (1998). Use of the handicap creation process model to explain perceptions of the barriers to work reintegration of persons with aphasia. Working paper, University of Ottawa, Faculty of Administration.

GETKATE, M., P.HAUSDORF ET S.F. CRONSHAW (1992). Transnational validity generalization of employment tests from the United States to Canada. Canadian Journal of Administrative Sciences, vol. 9, no 4, 324-335.

GORMAN, C.D., W.H. CLOVER ET M.E. DOHERTY (1978). «Can we learn anything about interviewing real people from interviewers of paper people? Two studies of the external validity paradigm», Organizational Behavior and Human Performance, no 22, 165-192.

HEILMAN, M.E. ET R.F. MARTELL (1986). Exposure to successful women: Antidote to sex discrimination in applicant screening decisions», Organizational Behavior and Human Decision Process, no 37, 376-390.

HÉTU,R.,L. GETTY ET S.WARIDEL (1994). «Attitudes towards co-workers affected by occupational hearing loss II: focus group interviews», British Journal of Audiology, no 28, 313-325.

JACKSON,D.N., C.P.PEACOCK ET J.P.SMITH (1980). «Impressions of personality in the employment interview» Journal of Personality and Social Psychology, vol. 39, no 2, 294-307.

JOHNSON, R. (1987). «Return to work after severe head injury». International Disability Studies, vol. 9, 49-54.

KALINOWSKI, J., J. ARMSON, A. STUART ET J.W. LERMAN (1993). «Speech clinicians and the general public's perceptions of self and stutterers», Journal of Speech-Language Pathology and Audiology, vol 7, no 2, 79-85. 
KUTSCHER, R.E. (1992). «Outlook 1990-2005: Major trends and issues», Occupational Outlook Quarterly, vol 36, no 1, 2-5.

KYLE,J.G.ET P.L.WOOD (1985). «Vocational aspects of acquired hearing loss», International Journal of Rehabilitation Research, vol. 8, 425-434.

LONDON, M. ET M.D. HAKEL (1974). «Effects of applicant stereotypes, order, and information on interview stereotypes», Journal of Applied Psychology, no 59, 157-162.

MACLEOD-GALLINGER, J.E. (1992). «The career status of deaf women: A comparative look». American Annals of the Deaf, no 137,315-325.

MCDONALD, T.ET M.D. HAKEL (1985). «Effects of applicant race, sex, suitability and answers on interviewers questioning strategy and rating», Personnel Psychology, no 38, 321-334.

MCKINNON, S.L., C.W. HESS ET R.G. LANDRY (1986). «Reactions of college students to speech disorders", Journal of Communication Disorders, no 19, 75-82.

NESTER, M.A. (1984). «Employment testing for handicapped persons», Public Personnel Management Journal, no 13, 417-434.

RICE, M.ET R. KROLL (1994). "A survey of stutterer's perceptions of challenges and discrimination in the work place», Proceedings of the First World Congress on Fluency Disorders, no 2, 559-562.

RICE, M. (1995). Work place experiences of people who stutter. Presented at the World Conference of the International Fluency Association, Linkoping, Sweden.

ROLLAND, J.ET C. BELIN (1991). «L'aphasique et le monde du travail», dans J. Ponzio, D. Lafond, R. Degiovani et Y. Joanette (éd.), L'apahasique, Montréal, Edisem, Inc. 195-211.

SHREY,D.E. (1997a). «Work-site disability management and industrial rehabilitation an overview», dans E. Donald, D.E. Shrey et Michel Lacerte, Principles and practice of disability management in industry. GR Press Inc., Florida.

STATISTICS CANADA (1990). Highlights: Disabled Persons in Canada. The Health and Activity Limitation Survey, Ottawa, Catalogue \# 82-602.

STATISTICS CANADA (1990). Highlights: Disabled Persons in Canada. The Health and Activity Limitation Survey, Ottawa, Catalogue \# 82-602.

THOMAS,A.ET K.G.HERBST (1980). «Social and psychological implications of acquired deafness for adults of employment age». British Journal of Audiology, vol.14, 76-85.

\section{Notes}

1. Selon Fougeyrollas et al. (1996), une incapacité correspond au degré de réduction d'une aptitude. Une aptitude est la possibilité pour une personne d'accomplir une activité physique ou mentale, sans tenir compte de l'environnement.

2. Le lecteur pourra trouver dans Garcia, Barrette et Laroche (1998) une description détaillée de la méthodologie utilisée dans cette recherche. 